\title{
Development and Validation of High Performance Liquid Chromatography Method for Simultaneous Estimation of Ambroxol and Doxofylline in Their Combined Tablet Dosage Form
}

\author{
Neha Singhal, ${ }^{1}$ Kalpesh Gaur, ${ }^{2}$ Anoop Singh, ${ }^{1}$ and Karni Singh Sekhawat ${ }^{1}$ \\ ${ }^{1}$ Vidyasthali Institute of Technology, Science and Management, Durgapura, Jaipur, Rajasthan 302018, India \\ ${ }^{2}$ Geetanjali Institute of Pharmacy, Geetanjali University, Udaipur, Rajasthan 313002, India
}

Correspondence should be addressed to Kalpesh Gaur; kalpeshgaur@gmail.com

Received 3 June 2013; Accepted 27 June 2013

Academic Editors: A. Bouklouze, D. Kara, and A. Orte

Copyright (C) 2013 Neha Singhal et al. This is an open access article distributed under the Creative Commons Attribution License, which permits unrestricted use, distribution, and reproduction in any medium, provided the original work is properly cited.

The present study described a new, simple, accurate, and precise high performance liquid chromatography method for the simultaneous determination of Ambroxol and Doxofylline in combined tablet dosage form. The chromatographic method was standardized using a BDS hypersil $\mathrm{C}_{18}, 250 \mathrm{~mm} \times 4.6 \mathrm{~mm}, 5 \mu$ (particle size), Thermo scientific from Germany with isocratic conditions, and mobile phase containing potassium dihydrogen orthophosphate buffer-pH $4.5\left(0.05 \mathrm{M} \mathrm{KH}_{2} \mathrm{PO}_{4}\right)$ : acetonitrile $(60: 40)$ at flow rate of $1 \mathrm{ml} / \mathrm{min}$ using UV detection at $254 \mathrm{~nm}$. The retention times of Ambroxol and Doxofylline were $3.510 \mathrm{~min}$ and $7.247 \mathrm{~min}$, respectively. The method was linear over the concentration range for Ambroxol 3.75-11.25 $\mu \mathrm{g} / \mathrm{mL}$ and for Doxofylline $50-150 \mu \mathrm{g} / \mathrm{mL}$. The recovery of Ambroxol and Doxofylline was found to be in the range of 99.42-101.18\% and 99.37-100.28\%, respectively. The validation of method was carried out using ICH guidelines. The described HPLC method was successfully employed for the analysis of pharmaceutical formulations containing combined dosage form.

\section{Introduction}

Ambroxol is a secretolytic agent used in the treatment of tracheobronchitis, emphysema with bronchitis pneumoconiosis, chronic inflammatory pulmonary conditions, bronchiectasis, and bronchitis with bronchospasm asthma [1]. Chemically, it is trans-4-[(2-Amino-3,5-dibromobenzyl)amino]cyclohexanol hydrochloride [2]. Literature survey revealed that few spectroscopic methods RP-HPLC, HPTLC, and UPLC [3-10] methods have been reported for the estimation of Ambroxol with other drugs.

Doxofylline is a new generation long acting oral methylxanthine derivative. Methylxanthines are phosphodiesterase inhibitors. It is mainly used for maintenance therapy in patients suffering from asthma and Chronic Obstructive Pulmonary Disease (COPD). Chemically, it is 7-(1,3-dioxolan-2-ylmethyl)-3,7-dihydro-1,3-dimethyl-1Hpurine-2,6-dione [11]. Literature survey revealed that few spectrophotometric methods and RP-HPLC method have been reported for the estimation of Doxofylline with other drugs [12-15].

From the literature survey, it was found that many methods have been reported for estimation of Ambroxol and Doxofylline individually and in combination with other drugs, and no HPLC method for simultaneous estimation of Ambroxol and Doxofylline has been reported so far. Hence an attempt has been made to develop new HPLC method which is simple, rapid, reproducible, and economical method for simultaneous estimation of Ambroxol and Doxofylline in tablet dosage form.

\section{Materials and Methods}

2.1. Chemicals and Reagents. The working standards of Ambroxol and Doxofylline were generous gift obtained from Ranbaxy Laboratories Ltd., India. The combination formulation of Ambroxol and Doxofylline (Synasma-AX) were 
marketed by Ranbaxy Laboratories Ltd., India; tablets were purchased from the local market. Acetonitrile, methanol, and water were used of HPLC grade make-Merck, Rankem. Potassium dihydrogen phosphate and phosphoric acid used were of analytical grade.

2.2. HPLC Instrumentation. Chromatographic separation was performed with Shimadzu Prominence System (SPD20AT, Shimadzu) having the following components: LC20AT Pump, SPD-20A Detector, BDS hypersil $\mathrm{C}_{18}, 250 \mathrm{~mm} \times$ $4.6 \mathrm{~mm}, 5 \mu$ (particle size), Thermo scientific, Rheodyne manual Injector (20 $\mu \mathrm{L}$ Capacity), Hamilton Syringe $(25 \mu \mathrm{L})$, and Chromatograms, and data were recorded by means of Spinchrom CFR Software.

\subsection{Preparation of Mobile Phase and Standard Solution}

2.3.1. Mobile Phase Preparation. The mobile phase consisted of potassium dihydrogen phosphate buffer $\mathrm{pH} 4.5$ acetonitrile $(60: 40)$. To prepare the buffer solution, $6.8 \mathrm{gm}$ potassium dihydrogen phosphate was weighed and dissolved in $900 \mathrm{~mL}$ HPLC grade water in $1000 \mathrm{~mL}$ volumetric flask. The $\mathrm{pH}$ of the buffer was adjusted to 4.5 with diluted phosphoric acid $\left(\mathrm{H}_{3} \mathrm{PO}_{4}\right)$. The volume is made up to $1000 \mathrm{~mL}$ with HPLC grade water. Mobile phase was filtered through a $0.45 \mu \mathrm{m}$ nylon membrane (Millipore Pvt. Ltd., Bangalore, India) and degassed in an ultrasonic bath (Toshcon by Toshniwal).

\subsubsection{Standard Preparation}

Stock Solution of Ambroxol. Ambroxol standard stock solution containing $75 \mu \mathrm{g} / \mathrm{mL}$ was prepared in a $100 \mathrm{~mL}$ volumetric flask by dissolving $7.5 \mathrm{mg}$ of Ambroxol in small volume of mobile phase; then make up the volume up to $100 \mathrm{~mL}$ with mobile phase. This solution is then sonicated for 10 minutes.

Stock Solution of Doxofylline. Doxofylline standard stock solution containing $1000 \mu \mathrm{g} / \mathrm{mL}$ was prepared in a $100 \mathrm{~mL}$ volumetric flask by dissolving $100 \mathrm{mg}$ of Doxofylline in small volume of mobile phase; then make up the volume up to $100 \mathrm{~mL}$ with mobile phase. This solution is then sonicated for 10 minutes.

Working Standard Preparation. Take $1 \mathrm{~mL}$ of Ambroxol stock solution and $1 \mathrm{~mL}$ of Doxofylline stock solution and dilute with mobile phase up to $10 \mathrm{~mL}$. Then solution was filtered through $0.45 \mu \mathrm{m}$ nylon syringe filter. The concentration obtained was $7.5 \mu \mathrm{g} / \mathrm{mL}$ of Ambroxol and $100 \mu \mathrm{g} / \mathrm{mL}$ of Doxofylline.

2.4. Chromatographic Conditions. The mobile phase consisting of phosphate buffer-pH $4.5\left(0.05 \mathrm{M} \mathrm{KH}_{2} \mathrm{PO}_{4}\right)$ : acetonitrile in the ratio $(60: 40)$ with an apparent $\mathrm{pH}$ adjusted to 4.5 using diluted phosphoric acid was selected as the optimum composition of mobile phase, because it was found that this solvent system resolved both the components ideally. The mobile phase and samples were degassed by ultrasonication for $20 \mathrm{~min}$ and filtered through $0.45 \mu \mathrm{m}$ Nylon 66 (N66) $47 \mathrm{~mm}$ membrane filter paper. The measurements were carried out with an injection volume of $20 \mu \mathrm{L}$, flow rate was set to $1.0 \mathrm{~mL} / \mathrm{min}$, and UV detection was carried out at $254 \mathrm{~nm}$. All determinations were done at ambient column temperature $\left(27^{\circ} \mathrm{C}\right)$. The chromatograms of the prepared standard stock solutions of Ambroxol and Doxofylline were recorded under the above optimized chromatographic conditions (Figure 3).

2.5. Test Preparation. Twenty tablets were weighed, and the average weight was calculated. The tablets were crushed with a mortar and pestle for $10 \mathrm{~min}$. A portion of powder equivalent to the weight of one tablet was accurately weighed and transferred to a $100 \mathrm{~mL}$ volumetric flask, diluted with mobile phase, and sonicated of 30 minutes with normal handshaking; then cool the flask to room temperature; afterward filter this solution through $0.45 \mu \mathrm{m}$ nylon syringe filter. The concentration obtained was $7.5 \mu \mathrm{g} / \mathrm{mL}$ of Ambroxol and $100 \mu \mathrm{g} / \mathrm{mL}$ of Doxofylline. The chromatogram of the prepared solution of Ambroxol and Doxofylline was recorded under the above optimized chromatographic conditions (Figure 4).

\section{Result and Discussion}

3.1. Method Development and Optimization. Proper selection of the methods depends upon the nature of the sample (ionic or ionisable or neutral molecule), its molecular weight, and solubility. Ambroxol and Doxofylline were dissolved in polar solvent, so the developed method of estimation was called as reverse phase high performance liquid chromatography. To develop a rugged and suitable HPLC method for the quantitative determination of Ambroxol and Doxofylline, the analytical condition was selected after the consideration of different parameters such as diluent, buffer, buffer concentration, organic solvent for mobile phase and mobile phase composition, and other chromatographic conditions. Preliminary trials were taken with different composition of buffer and organic phase of mobile phases with $\mathrm{pH}$ range of 3-5. The column selection has been done by backpressure, resolution, peak shape, theoretical plates, and day-to-day reproducibility of the retention time and resolution between Ambroxol and Doxofylline peak. After evaluating all these factors, a BDS hypersil $\mathrm{C}_{18}$ column was found to be giving satisfactory results. The selection of acetonitrile and buffer were based on chemical structure of both the drugs (Figures 1 and 2). The acidic $\mathrm{pH}$ range was found suitable for solubility, resolution, stability, theoretical plates, and peak shape of both components. Best results were obtained with $0.05 \mathrm{M}$ potassium dihydrogen orthophosphate $\mathrm{pH} 4.5$ with phosphoric acid solution that improved the peak shapes of Ambroxol and Doxofylline. For the selection of organic constituent of mobile phase, acetonitrile was chosen to reduce the longer retention time and to attain good peak shape. Therefore, final mobile phase composition consisting of a mixture of buffer-pH $4.5\left(0.05 \mathrm{M} \mathrm{KH}_{2} \mathrm{PO}_{4}\right)$ : acetonitrile (60:40), set at a flow rate of $1.0 \mathrm{~mL} / \mathrm{min}$ was selected for the chromatographic analysis. Optimized mobile phase proportion was providing good resolution between Ambroxol and Doxofylline. 
<smiles>Nc1c(Br)cc(Br)cc1CN[C@H]1CC[C@H](O)CC1</smiles>

FIGURE 1: Chemical structure of Ambroxol.

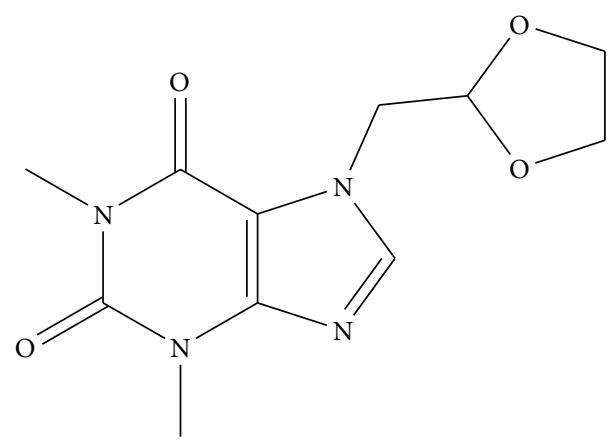

Figure 2: Chemical structure of Doxofylline.

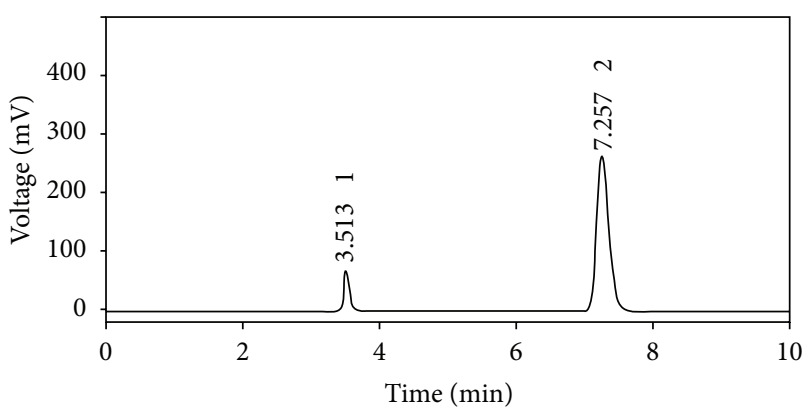

Figure 3: HPLC chromatogram of Ambroxol and Doxofylline standards.

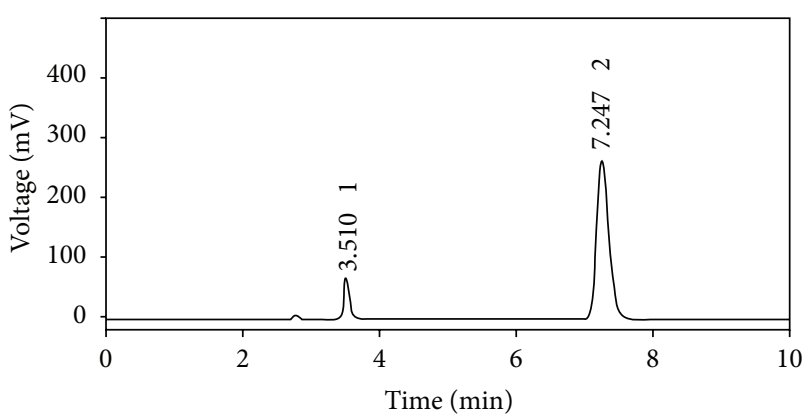

FIgURE 4: HPLC chromatogram of Ambroxol and Doxofylline in tablet formulation.

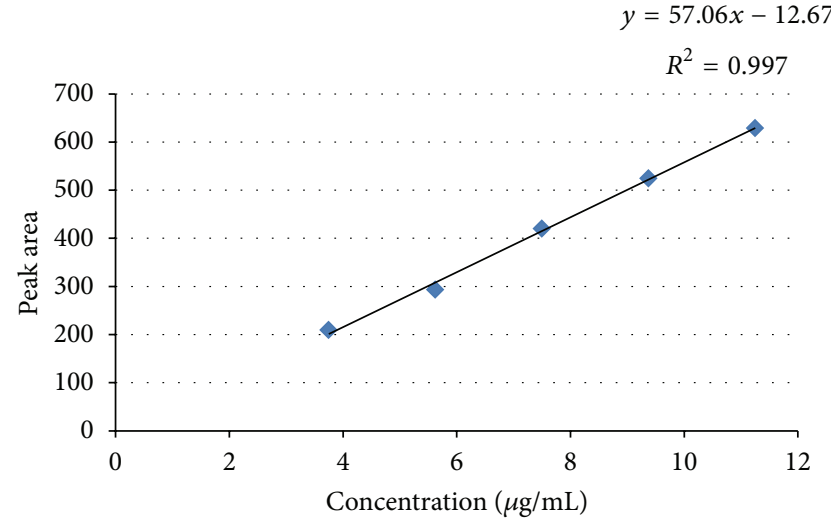

FIgURE 5: Linearity study for Ambroxol.

Under above described experimental conditions, all the peaks were well defined and free from tailing. The concern of small deliberate changes in the mobile phase composition, flow rates, and column temperature on results was evaluated as a part of testing for methods robustness. The peak homogeneity was expressed for peak purity values and was obtained directly from the spectral analysis of the sample.

3.2. Method Validation. The developed analytical method was subjected to validation with respect to various parameters such as linearity, limit of quantification (LOQ), limit of detection (LOD), accuracy, precision, recovery studies, specificity and reproducibility, and robustness/ruggedness as per the ICH guidelines [16-21] (Table 5).

3.2.1. Specificity. The specificity of the method was evaluated by assessing interference from excipients in the pharmaceutical dosage form prepared as a placebo solution. The specificity of the method for the drug was also established by checking for interference with drug quantification from degradation products formed during the forced degradation study. The peak purity of the Ambroxol and Doxofylline was found satisfactory under different stress conditions. There was no interference of any peak of degradation product with drug peak.

3.2.2. Linearity and Range. For linearity, five-point calibration curve was obtained in a concentration range from 3.75 to $11.25 \mu \mathrm{g} / \mathrm{mL}$ for Ambroxol and $50-150 \mu \mathrm{g} / \mathrm{mL}$ for Doxofylline. The response of the drug was found to be linear in the investigation concentration range, and the linear regression equation for Ambroxol was $y=57.06 x-12.67$ with correlation coefficient 0.997 (Figure 5) and for Doxofylline was $y=34.24 x-60.39$ with correlation coefficient 0.998 (Figure 6) where $x$ is the concentration in $\mu \mathrm{g} / \mathrm{mL}$ and $y$ is the peak area in absorbance unit.

3.2.3. Precision (Repeatability and Reproducibility). Precision study was established by evaluating method precision and intermediate precision study. System precision was evaluated 
TABLE 1: Precision data for Ambroxol and Doxofylline.

\begin{tabular}{|c|c|c|c|c|c|}
\hline Drug & Concentration $(\mu \mathrm{g} / \mathrm{mL})$ & Intraday $(n=3)$ average \pm SD & C.V. & Interday $(n=3)$ average \pm SD & C.V. \\
\hline \multirow{3}{*}{ Ambroxol } & 3.75 & $209.47 \pm 1.71$ & 0.82 & $209.88 \pm 2.29$ & 1.09 \\
\hline & 7.50 & $419.91 \pm 4.43$ & 1.05 & $421.32 \pm 4.84$ & 1.15 \\
\hline & 11.25 & $629.12 \pm 6.11$ & 0.97 & $627.44 \pm 5.84$ & 0.93 \\
\hline \multirow{3}{*}{ Doxofylline } & 50 & $1689.00 \pm 16.78$ & 0.99 & $1691.66 \pm 14.54$ & 0.86 \\
\hline & 100 & $3384.19 \pm 40.70$ & 1.20 & $3396.37 \pm 35.26$ & 1.04 \\
\hline & 150 & $5067.86 \pm 38.96$ & 0.77 & $5055.92 \pm 55.21$ & 1.090 \\
\hline
\end{tabular}

TABLE 2: Results of accuracy study.

\begin{tabular}{|c|c|c|c|c|c|c|}
\hline Drug & Label claim & Result & $\%$ assay & Average $\%$ assay & SD & $\%$ RSD \\
\hline \multirow{3}{*}{ Ambroxol } & 7.5 & 7.36 & 98.09 & \multirow{3}{*}{98.42} & \multirow{3}{*}{0.303337} & \multirow{3}{*}{0.308202} \\
\hline & 7.5 & 7.39 & 98.48 & & & \\
\hline & 7.5 & 7.40 & 98.69 & & & \\
\hline \multirow{3}{*}{ Doxofylline } & 100 & 98.11 & 98.11 & \multirow{3}{*}{98.19} & \multirow{3}{*}{0.47832} & \multirow{3}{*}{0.487141} \\
\hline & 100 & 97.75 & 97.75 & & & \\
\hline & 100 & 98.70 & 98.70 & & & \\
\hline
\end{tabular}

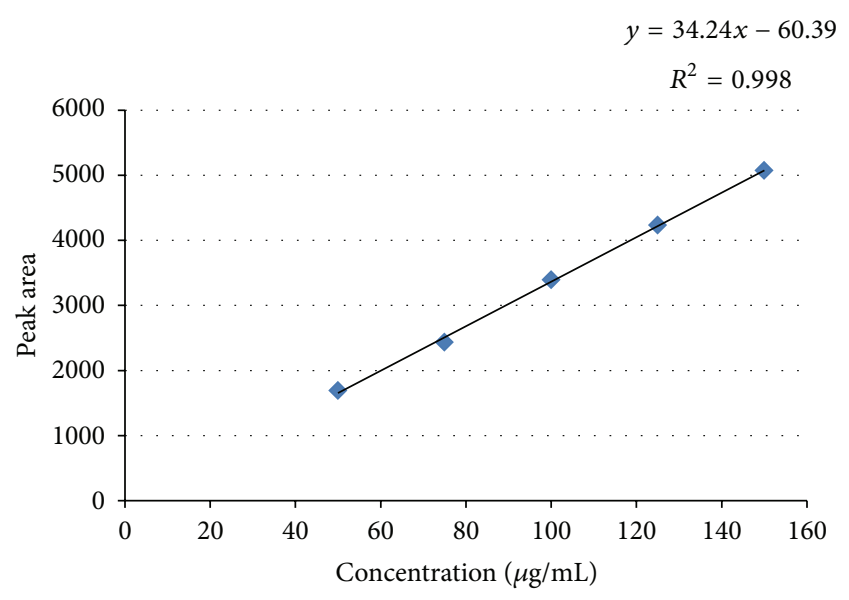

FIgURE 6: Linearity study for Doxofylline.

TABLE 3: Results of recovery study of Ambroxol.

\begin{tabular}{lccc}
\hline $\begin{array}{l}\text { Concentration } \\
\text { level }\end{array}$ & $\begin{array}{c}\text { Standard: amount } \\
\text { added in sample } \\
\text { solution in } \mu \mathrm{g} / \mathrm{mL}\end{array}$ & $\begin{array}{c}\text { Amount } \\
\text { recovered } \\
\text { in } \mu \mathrm{g} / \mathrm{mL}\end{array}$ & \% recovery \\
\hline $80 \%$ & 6 & 5.98 & 99.71 \\
$80 \%$ & 6 & 6.03 & 100.57 \\
$80 \%$ & 6 & 6.00 & 100.04 \\
$100 \%$ & 7.5 & 7.49 & 99.84 \\
$100 \%$ & 7.5 & 7.56 & 100.84 \\
$100 \%$ & 7.5 & 7.53 & 100.34 \\
$120 \%$ & 9 & 8.95 & 99.43 \\
$120 \%$ & 9 & 9.11 & 101.18 \\
$120 \%$ & 9 & 9.01 & 100.14 \\
\hline
\end{tabular}

Note: sample concentration was used $7.5 \mu \mathrm{g} / \mathrm{mL}$.

by analyzing the standard solution five times. Method precision of the analytical method was determined by analyzing
TABLE 4: Results of recovery study of Doxofylline.

\begin{tabular}{lccc}
\hline $\begin{array}{l}\text { Concentration } \\
\text { level }\end{array}$ & $\begin{array}{c}\text { Standard: amount } \\
\text { added in sample } \\
\text { solution in } \mu \mathrm{g} / \mathrm{mL}\end{array}$ & $\begin{array}{c}\text { Amount } \\
\text { recovered } \\
\text { in } \mu \mathrm{g} / \mathrm{mL}\end{array}$ & \% recovery \\
\hline $80 \%$ & 80 & 79.72 & 99.65 \\
$80 \%$ & 80 & 79.69 & 99.62 \\
$80 \%$ & 80 & 79.98 & 99.97 \\
$100 \%$ & 100 & 99.78 & 99.78 \\
$100 \%$ & 100 & 99.60 & 99.60 \\
$100 \%$ & 100 & 100.28 & 100.28 \\
$120 \%$ & 120 & 119.25 & 99.37 \\
$120 \%$ & 120 & 119.94 & 99.95 \\
$120 \%$ & 120 & 120.11 & 100.09 \\
\hline
\end{tabular}

Note: sample concentration was used $100 \mu \mathrm{g} / \mathrm{mL}$.

TABLE 5: Statistical data for Ambroxol and Doxofylline by HPLC method.

\begin{tabular}{lcc}
\hline Parameter & Ambroxol & Doxofylline \\
\hline Linear range $(\mu \mathrm{g} / \mathrm{mL})$ & $3.75-11.25$ & $50-150$ \\
Slope & 57.06 & 34.24 \\
Limit of detection $(\mu \mathrm{g} / \mathrm{mL})$ & 0.59 & 1.80 \\
Limit of quantitation $(\mu \mathrm{g} / \mathrm{mL})$ & 4.97 & 15.06 \\
\hline
\end{tabular}

three sets of sample preparation. Assay of all three replicate sample preparations was determined and standard deviation; $\%$ relative standard deviation was calculated.

Intermediate precision of the analytical method was determined by performing method precision on another day by another analyst under the same experimental condition. Assay of all three replicate sample preparations was determined and standard deviation; \% relative standard deviation was calculated. 
TABle 6: Robustness data for Ambroxol.

\begin{tabular}{lcccc}
\hline Factors & \% RSD & Tailing factor $\left(A_{S}\right)$ & Efficiency (theoretical plates) & Resolution \\
\hline pH of mobile phase & & & & \\
pH 4.3 & 1.1935 & 1.455 & 6903 & 14.724 \\
pH 4.7 & 0.7549 & 1.348 & 7318 & 14.810 \\
\hline Flow rate & & & & 14.736 \\
$0.8 \mathrm{~mL} / \mathrm{min}$ & 0.7022 & 1.375 & 7090 & 14.745 \\
\hline $.2 \mathrm{~mL} / \mathrm{min}$ & 0.7072 & 1.429 & 7109 & 14.698 \\
\hline Solvent \% & & & & 6890 \\
$-2 \%$ & 1.0015 & 1.348 & 6851 & 14.672 \\
\hline $2 \%$ & 1.1029 & 1.455 & & \\
\hline
\end{tabular}

TABLE 7: Robustness data for Doxofylline.

\begin{tabular}{lcccc}
\hline Factors & \% RSD & Tailing factor $\left(A_{S}\right)$ & Efficiency (theoretical plates) & Resolution \\
\hline pH of mobile phase & & & & \\
pH 4.3 & 1.3415 & 1.362 & 7347 & 14.724 \\
pH 4.7 & 0.9134 & 1.391 & 7273 & 14.810 \\
\hline Flow rate & & & & 14.736 \\
$0.8 \mathrm{~mL} / \mathrm{min}$ & 0.5180 & 1.367 & 7294 & 14.745 \\
\hline $.2 \mathrm{~mL} / \mathrm{min}$ & 0.8075 & 1.386 & 7292 & 14.698 \\
\hline Solvent \% & & & & 14.672 \\
$-2 \%$ & 1.1918 & 1.348 & 7293 & \\
$+2 \%$ & 0.9294 & 1.413 & & 7293 \\
\hline
\end{tabular}

Data obtained from precision experiments are given in Table 1 for intraday and interday precision study for both Ambroxol and Doxofylline. The RSD values for intraday precision study and interday precision study were $<2.0 \%$ for Ambroxol and Doxofylline that confirm the method was precise.

3.2.4. Accuracy (\% Assay). Accuracy study was assessed by determination of the \% assay of Ambroxol and Doxofylline in market formulation. The mean \% assay of Ambroxol was $98.42 \%$, and the mean recovery of Doxofylline was $98.18 \%$, that was satisfactory (Table 2).

3.2.5. Recovery Studies. Recovery of Ambroxol and Doxofylline was done at three different concentrations (corresponding to 80,100 , and $120 \%$ of test solution concentration). Known amounts of Ambroxol $(3.75,7.5$, and $11.25 \mu \mathrm{g} / \mathrm{mL})$ and Doxofylline $(50,100$, and $150 \mu \mathrm{g} / \mathrm{mL})$ were added to a diluent preparation, and the amount of Ambroxol and Doxofylline recovered was calculated. For each concentration, three sets were prepared and injected in duplicate. \% recovery was calculated at each level and recorded as shown in Tables 3 and 4. The mean recovery of Ambroxol was between $99.42 \%$ and $101.18 \%$, and the mean recovery of Doxofylline was between $99.37 \%$ and $100.28 \%$, which was satisfactory.

3.2.6. Limit of Detection ( $L O D) /$ Limit of Quantitation (LOQ). The LOD was determined on the basis of signal to noise ratios and was determined using analytical response of three times the background noise. LOQ was determined as the lowest amount of analyte that was reproducibly quantified above the baseline noise following triplicate injections. Both LOQ and LOD were calculated on the peak area using the following equations:

$$
\begin{aligned}
& \mathrm{LOQ}=10 \times \frac{N}{B}, \\
& \mathrm{LOD}=3 \times \frac{N}{B},
\end{aligned}
$$

where $N$ is the standard deviation (SD) of the peak areas (triplicate injections) of the drug and $B$ is the slope of the corresponding calibration curve.

The limit of detection and limit of quantification were evaluated by serial dilutions of Ambroxol and Doxofylline stock solution in order to obtain signal to noise ratio of $3: 1$ for LOD and 10:1 for LOQ. The LOD value for Ambroxol and Doxofylline was found to be $0.597 \mu \mathrm{g} / \mathrm{mL}$ and $1.809 \mu \mathrm{g} / \mathrm{mL}$, respectively, and the LOQ value $4.970 \mu \mathrm{g} / \mathrm{mL}$ and $15.061 \mu \mathrm{g} / \mathrm{mL}$, respectively.

3.2.7. Robustness. The robustness was studied by evaluating the effect of small but deliberate variations in the chromatographic conditions. The conditions studied were flow rate (altered by $\pm 0.2 / \mathrm{min}$ ), mobile phase composition (by using $58: 42$ and $62: 38 \mathrm{v} / \mathrm{v}$ buffer $\mathrm{pH} 4.5$ : acetonitrile), buffer $\mathrm{pH}$ (altered by \pm 0.2 ), and use of HPLC columns from different batches. The result of robustness study of the developed assay method was established in Tables 6 and 7. The result shown that during all variance conditions, assay value of the test preparation solution was not affected and it was in accordance 
TABLE 8: System suitability test parameter.

\begin{tabular}{lcc}
\hline System suitability parameters & Ambroxol & Doxofylline \\
\hline Retention times $\left(R_{T}\right)(\min )$ & 3.510 & 7.247 \\
Theoretical plates $(N)$ & 7318 & 7293 \\
Resolution $\left(R_{S}\right)$ & & 14.850 \\
Tailing factor $\left(A_{S}\right)$ & 1.455 & \\
\hline
\end{tabular}

with that of actual. System suitability parameters were also found satisfactory; hence the analytical method would be concluded as robust.

3.2.8. System Suitability. The system suitability tests represent an integral part of the method and are used to ensure adequate performance of the chromatographic system. The parameters, retention time $\left(R_{T}\right)$, theoretical plates $(N)$, peak resolution $(R)$, peak asymmetry $(T)$, and repeatability were evaluated using five replicate injections of the drugs. Acceptance criteria for system suitability are as follows: asymmetry should not be more than 2.0, resolution should not be more than 2.0, theoretical plate should not be less than 6800 , and $\%$ RSD of peak area should not be more than 2.0 as per the results of present study, and the outcomes of validation studies were shown as the good results during all validation parameters. The result of system suitability study of the developed assay method was shown in Table 8.

The surveillance and results obtained from each validation experiment including specificity, linearity and range, LOD and LOQ, precision, accuracy, robustness, recovery, and system suitability lie well inside the acceptance criteria of ICH guideline. Since all the results are within the limit, the developed analytical method is considered as validated and suitable for probable use.

\section{Conflict of Interests}

The authors wish to confirm that there is no known conflict of interests associated with this paper. The authors confirm that they have given due consideration to the protection of intellectual property associated with this work and that there is no impediment to publication, including the trademarks mentioned in their paper.

\section{Acknowledgment}

The authors are heartily grateful to Mr. Ketan Patel, Director, Molecule Laboratory, Ahmedabad, Gujarat, for providing all the facilities to carry out the research work.

\section{References}

[1] K. D. Tripathi, Essentials of Medical Pharmacology, Jaypee Brothers Medical Publishers, New Delhi, India, 6th edition, 2010.

[2] British Pharmacopoeia, vol. 2, The Department of Health: The Stationary Office, London, UK, 2009.
[3] M. Avhad and C. G. Bonde, "Development and validation of simultaneous uvspectrophotometric method for the determination of levofloxacin and ambroxol in tablets," International Journal of ChemTech Research, vol. 1, no. 4, pp. 873-888, 2009.

[4] P. S. Lakshmana, M. Srinivasan, S. Thiagarajan, and Q. Marina, "Simultaneous determination of gatifloxacin and ambroxol hydrochloride in a tablet formulation by liquid chromatography," Pharmaceutica Analytica Acta, vol. 1, no. 2, pp. 1-3, 2010.

[5] D. S. Nikam and S. C. Aswale, "Stability indicating RP-HPLC method for simultaneous estimation of ambroxol hydrochloride and roxithromycin in bulk and tablet dosage form," International Journal of Pharma Research and Development, vol. 2, no. 10, pp. 87-92, 2012.

[6] M. Sudhir, B. N. N. Rao, D. H. H. Theja, M. S. Prakash, P. Ramalingam, and A. M. Mohan Der, "Development of stability indicating RP-HPLC method for simultaneous determination of azithromycin and ambroxol $\mathrm{HCl}$ (SR) in the tablet formulation," Der Pharmacia Lettre, vol. 4, no. 3, pp. 803-810, 2012.

[7] M. V. Dhoka and S. S. Chopade, "Method development \& comparative statistical evaluation of HPLC \& HPTLC method for simultaneous estimation of cefodrixil monohydrate \& ambroxol hydrochloride," Indo Global Journal of Pharmaceutical Sciences, vol. 2, no. 2, pp. 203-212, 2012.

[8] A. B. Patel, S. G. Patel, D. P. Patel, B. H. Patel, and M. M. Patel, "Stability indicating HPTLC method development and validation for estimation of ambroxol hydrochloride and cetrizine dihydrochloride in combined tablet dosage form," International Research Journal of Pharmacy, vol. 2, no. 3, pp. 9599, 2011.

[9] E. A. Sharma and N. J. Shah, "Development and validation of high performance thin layer chromatography method for simultaneous estimation of ambroxol hydrochloride and desloratidine hydrochloride in their combined tablet dosage form," International Research Journal of Pharmacy, vol. 3, no. 5, pp. 305-308, 2012.

[10] R. K. Trivedi, M. C. Patel, and S. B. Jadhav, "A rapid, stability indicating RP-UPLC method for simultaneous determination of ambroxol hydrochloride, cetirizine hydrochloride and antimicrobial preservatives in liquid pharmaceutical formulation," Scientia Pharmaceutica, vol. 79, no. 3, pp. 525-543, 2011.

[11] Indian Pharmacopoeia, vol. III, Government of India, Ministry of Health and Family Welfare. The Indian Pharmacopoeia Commission, Ghaziabad, India, 2010.

[12] M. M. Kamila, N. Mondal, and L. K. Ghosh, "Development and validation of spectrophotometric method for estimation of anti-asthmatic drug doxofylline in bulk and pharmaceutical formulation," Indian Journal of Chemical Technology, vol. 14, no. 5, pp. 523-525, 2007.

[13] D. K. Jain, P. Patel, A. Kushwaha, R. S. Raghuwanshi, and N. Jain, "Simultaneous determination of Salbutamol sulphate and doxophylline in tablets by reverse phase liquid chromatography," Der Pharmacia Lettre, vol. 3, no. 4, pp. 56-62, 2011.

[14] A. Mittal and S. Parmar, "Development and validation of rapid HPLC method for determination of doxofylline in bulk drug and pharmaceutical dosage forms," Journal of Analytical Chemistry, vol. 65, no. 3, pp. 293-297, 2010.

[15] N. G. Patre, L. Sathiyanarayanan, M. V. Mahadik, and S. R. Dhaneshwar, "A validated, stability-indicating HPTLC method for analysis of doxofylline," Journal of Planar Chromatography, vol. 22, no. 5, pp. 345-348, 2009.

[16] L. R. Snyder, J. L. Glajch, and J. J. Kirkland, Practical HPLC Method Development, John Wiley \& Sons, 2nd edition, 1997. 
[17] A. H. Beckett and J. B. Stenlake, Practical Pharmaceutical Chemistry, part 2, CBS Publishers and Distributors, New Delhi, India, 4th edition, 1997.

[18] P. D. Sethi, HPLC: Quantitative Analysis of Pharmaceutical Formulation, CBS Publications, , New Delhi, India, 1996.

[19] ICH, Q2 (R1), Validation of Analytical Procedures: Text and Methodology, 2005.

[20] US FDA, Technical Review Guide: Validation of Chromatographic Methods, 1993.

[21] ICH Harmonized Tripartite Guideline, Validation of Analytical Procedure Methodology, Q2B, pp. 1-8, 1996. 

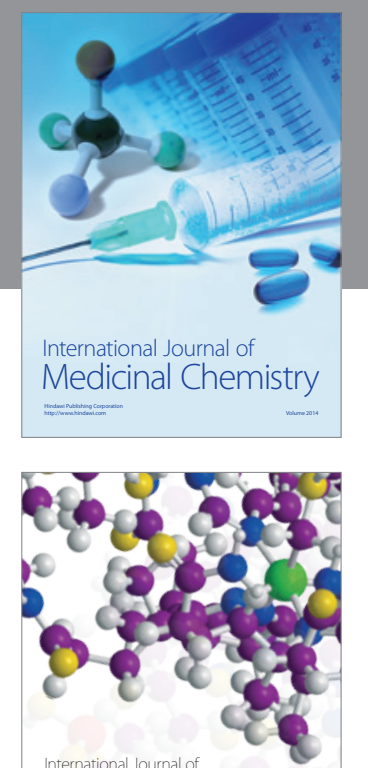

\section{Carbohydrate} Chemistry

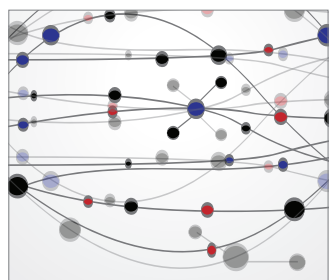

The Scientific World Journal
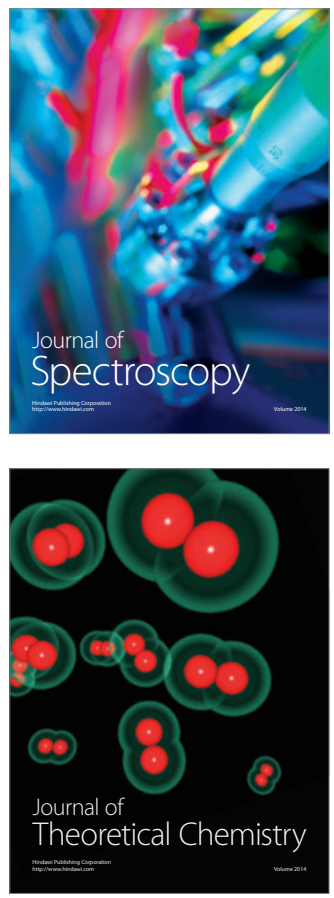
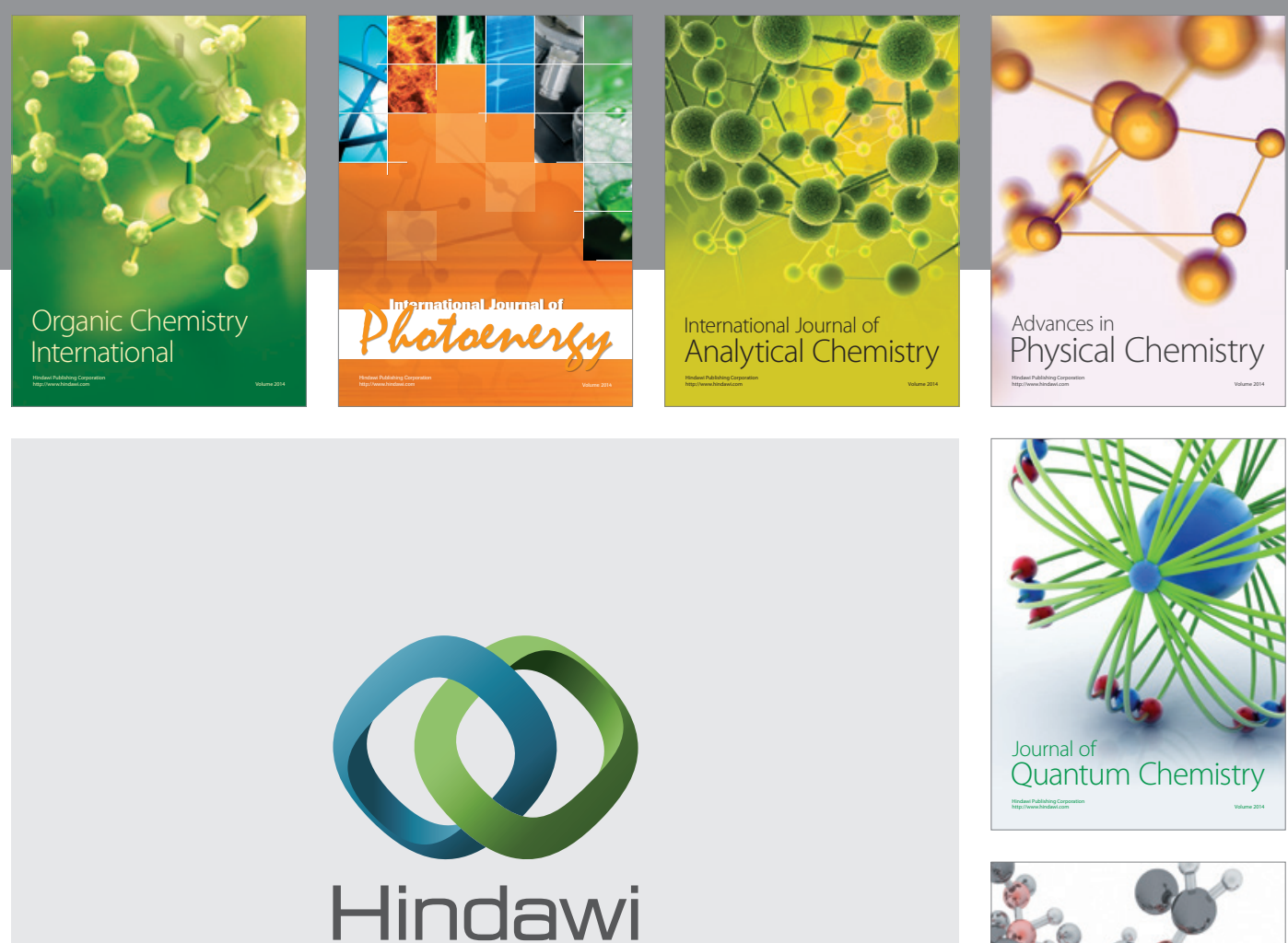

Submit your manuscripts at

http://www.hindawi.com

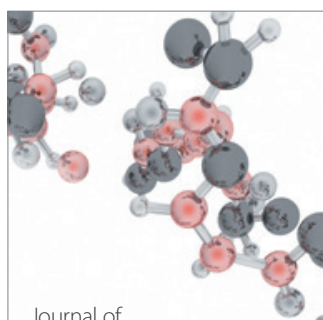

Analytical Methods

in Chemistry

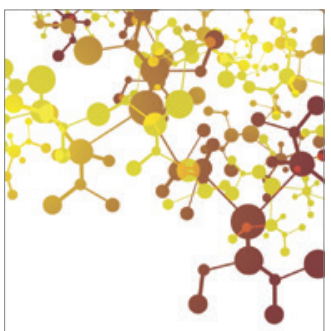

Journal of

Applied Chemistry

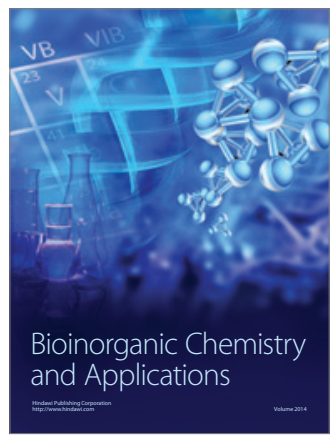

Inorganic Chemistry
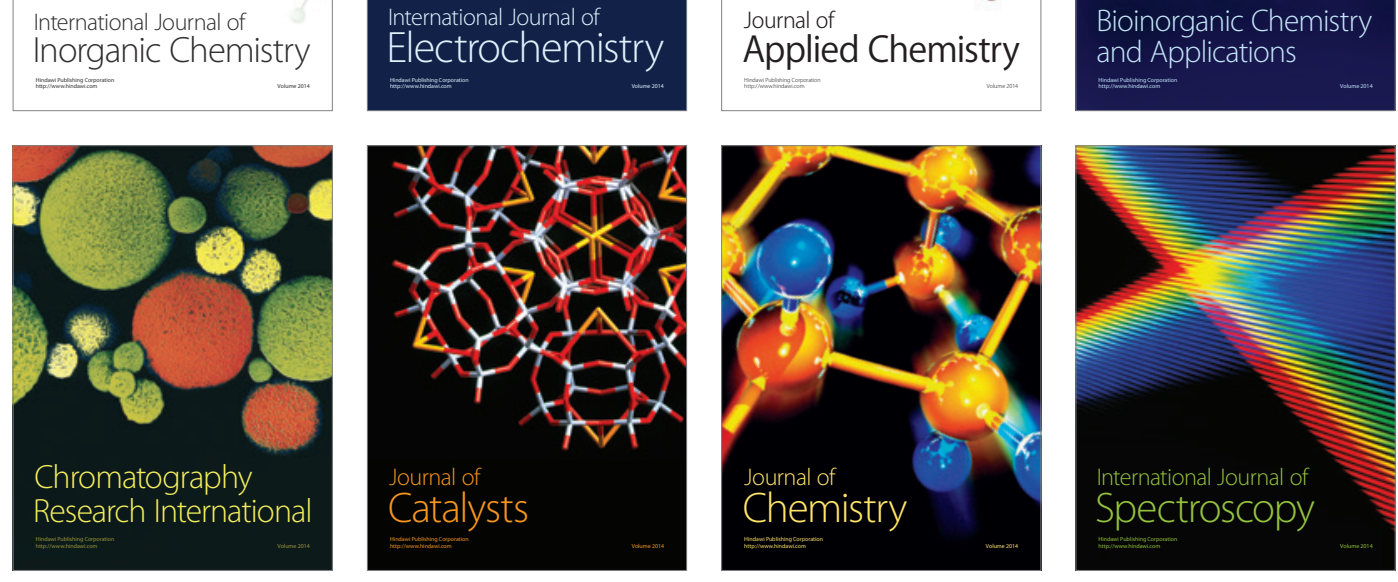